\title{
An IMT-Type Double Exponential Formula for Numerical Integration
}

By

\author{
Masatake MORI*
}

\begin{abstract}
A quadrature formula for evaluation of improper integrals over $(-1,1)$ is presented, which is obtained in such a way that the interval of integration $(-1,1)$ is transformed into $(-\infty, \infty)$ by $x=\tanh \left(A \sinh 2 B u /\left(1-u^{2}\right)\right)$ and that the trapezoidal rule with an equal mesh size is subsequently applied to the transformed integral. Asymptotic error analysis is made by means of the method of contour integral and a comparison with the IMTrule and the double exponential formula is given along with some numerical examples.
\end{abstract}

\section{§1. The IMT-rule and the Double Exponential Formula}

In 1969 Iri, Moriguti and Takasawa [4] presented a formula for numerical integration which is useful in particular in evaluating integrals with end point singularities. Their idea is to apply a variable transform to the given integral such that the function values of the transformed integrand vanish at the both end points of the transformed integral together with all its derivatives. This formula is known as the IMT-rule [2, p.114] and is thought to be one of the most efficient formulas for integrands with end point singularities [3].

On the other hand, Takahasi and Mori [9] proposed a family of formulas using a different kind of variable transform based on the asymptotic optimality of the trapezoidal rule for integrals over the infinite interval $(-\infty, \infty)[8$, pp. 74-76]. Consider an integral

$$
I=\int_{a}^{b} f(x) d x
$$

Received September 29, 1977. Revised November 24, 1977.

* Research Institute for Mathematical Sciences, Kyoto University, Kyoto 606, Japan. 
where $f(x)$ is assumed to be analytic on $(a, b)$. Then take a monotone increasing analytic function $\phi(u)$ which maps $(-\infty, \infty)$ onto $(a, b)$. Carrying out the variable transformation $x=\phi(u)$, we have

$$
I=\int_{-\infty}^{\infty} f(\phi(u)) \phi^{\prime}(u) d u
$$

Application of the trapezoidal rule with an equal mesh size $h$ leads to a formula

$$
I_{h}=h \sum_{n=-\infty}^{\infty} f(\phi(n h)) \phi^{\prime}(n h) .
$$

By choosing appropriate functions for $\phi(u)$ we obtain a variety of formulas. Similar investigations have been made by Schwartz [6] and Stenger [7], and by Sag and Szekeres [5] for multi-dimensional integrals.

Since $\left|f(\phi(n h)) \phi^{\prime}(n h)\right|$ decays rapidly as $|n| \rightarrow \infty$ by suitable choice of $\phi(u)$, we truncate the infinite summation (1.3) into a finite one, i. e. we replace (1.3) by

$$
I_{h}^{(N)}=h \sum_{n=-N^{-}}^{N^{+}} f(\phi(n h)) \phi^{\prime}(n h), N=N^{+}+N^{-}-1 .
$$

It is evident that, in error analysis, one should take into account both the discretization error

$$
\Delta I_{h}=I-I_{h}
$$

and the truncation error

$$
\varepsilon_{t}=I_{h}-I_{h}^{(N)}
$$

produced by truncation of the infinite summation, and the total error should be expressed in terms of the number $N$ of the sampling points actually used. Then it has been shown [10] that the mapping $x=\phi(u)$ is optimal with respect to the economy of $N$, i. e. the highest precision is obtained with the least number of function evaluations, when the transformed integrand behaves asymptotically in a double exponential way, i. e.

$$
\left|f(\phi(u)) \phi^{\prime}(u)\right| \approx \exp (-C \exp |u|), u \rightarrow \pm \infty,
$$


where $C$ is some positive constant. A formula constructed in this fashion is called a double exponential formula. In the case, for example, where the given integral is

$$
I=\int_{-1}^{1} f(x) d x
$$

the transformation

$$
x=\tanh \left(\frac{\pi}{2} \sinh u\right)
$$

gives a double exponential formula

$$
I_{h}=\frac{\pi}{2} h \sum_{n=-\infty}^{\infty} f\left(\tanh \left(\frac{\pi}{2} \sinh n h\right)\right) \frac{\cosh n h}{\cosh ^{2}\left(\frac{\pi}{2} \sinh n h\right)} .
$$

Although the IMT-rule has an advantage that it has no truncation error $\varepsilon_{t}$ mentioned above, it is rather difficult to compute the sampling points since each of them is defined by an integral of exponential function, while it is easy to compute the points and the weights of the double exponential formula, e.g. those of (1.10). Furthermore, it is shown that the asymptotic error expressions of the IMT-rule and the double exponential formula in terms of the number $N$ of the sampling points actually used are given as

$$
\left|\Delta I_{I M T}\right| \approx \exp \left(-C_{1} \sqrt{N}\right)
$$

and

$$
\left|\Delta I_{D E F}\right| \approx \exp \left(-C_{2} N / \log N\right),
$$

respectively, so that for large $N$ the latter is much better than the former.

\section{§2. An IMT-Type Double Exponential Formula}

It is natural to ask if there exists a formula which has both merits of the IMT-rule and of the double exponential formula, i. e. in which the number of points is finite, the points and the weights are easy to compute and the asymptotic error term behaves approximately as (1.12). The answer is affirmative, although there is a slight difference 
in the asymptotic error expressions.

Consider an integral

$$
I=\int_{-1}^{1} f(x) d x
$$

where $f(x)$ is analytic in a certain domain in the complex plane including the line segment $(-1,1)$ except possibly at $x= \pm 1 . \quad f(x)$ may have algebraic or logarithmic singularities at $x= \pm 1$ provided that it is integrable. Then the transforming function

$$
x=\phi(u)=\tanh \left(A \sinh B\left(\frac{1}{1-u}-\frac{1}{1+u}\right)\right),
$$

where $A$ and $B$ are certain positive constants, maps $(-1,1)$ onto itself and gives

$$
I=\int_{-1}^{1} g(u) d u
$$

where

$$
g(u)=f(\phi(u)) \phi^{\prime}(u) .
$$

Dividing $(-1,1)$ into $N$ equal subintervals and applying the trapezoidal rule, we have a formula

$$
I_{N}=\frac{2}{N} \sum_{n=1}^{N-1} f(\phi(-1+n h)) \phi^{\prime}(-1+n h), \quad h=\frac{2}{N} .
$$

This formula is similar to the IMT-rule in the sense that the function values of the transformed integrand together with all its derivatives vanish at $u= \pm 1$ and that the number of points is finite, while it is similar to the double exponential formula in the sense that the decay of $\left|f(\phi(u)) \phi^{\prime}(u)\right|$ as $u \rightarrow \pm 1$ is approximately double exponential. Hence we call the formula (2.5) obtained by (2.2) the IMT-type double exponential formula. It is evident that one can compute the points and the weights easily using exponential function.

\section{§ 3. Intrinsic Error of the Formula}

We shall give here an asymptotic error analysis of (2.5) briefly 
by means of the contour integral method. Since $f(z), z=x+i y$ is assumed to be analytic in the neighborhood of $(-1,1)$ except possibly at $z= \pm 1, g(w), w=u+i v$ of (2.4) is also analytic in the neighborhood of $(-1,1)$ except at $w= \pm 1$, so that the discretization error

$$
\Delta I_{N}=I-I_{N}
$$

can be expressed in terms of a contour integral as follows :

$$
\Delta I_{N}=\frac{1}{2 \pi i} \oint_{C} \hat{\Phi}_{N}(w) g(w) i w,
$$

where the path $C$ is a curve shown in Fig. 1 and is taken in such a way that there exists no singular point of $g(w)$ inside itself. When $N$ is sufficiently large, the characteristic function $\hat{\Phi}_{N}(w)$ of the error [8] is given approximately by

$$
\hat{\Phi}_{N}(w) \approx \pm 2 \pi i \exp ( \pm i \pi N w), \operatorname{Im} w \gtrless 0
$$

except in the close neighborhood of $w= \pm 1$ where the end point effect cannot be neglected $[1,8]$.

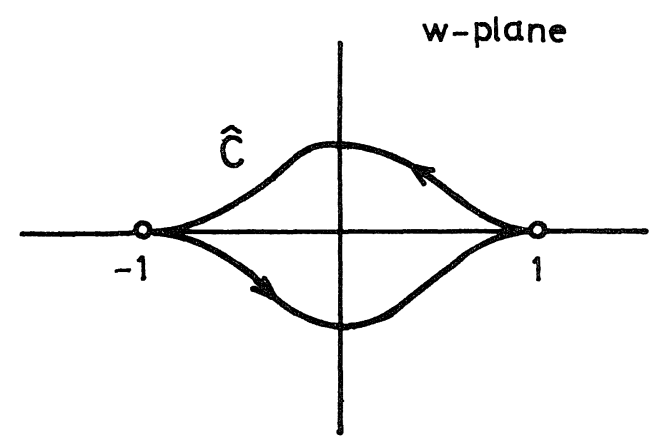

Fig. 1

Formulas obtained by a nonlinear transform usually do not integrate constants exactly, and the error produced when integrating a constant function can be regarded to be intrinsic to each formula. We generalize the idea of the intrinsic error of the present formula by defining it as the discretization error $\Delta I_{N}$ which is produced when we integrate

$$
f(x)=\left(1-x^{2}\right)^{\alpha}, \alpha>-1 .
$$


Now we shall derive an asymptotic representation for this intrinsic error from (3.2) by means of the saddle point method. For a general integrand that has singularities in the finite $z$-plane, the error other than the intrinsic one can be estimated by making use of the level map of the modulus of the characteristic function $\Phi_{N}(z)$ as we shall discuss in the next section.

If we substitute (2.2) and (3.4) into (2.4), we have

$$
g(w)=A B \cosh ^{-2(1+\alpha)}\left(A \sinh \left(\frac{2 B w}{1-w^{2}}\right)\right) \cosh \left(\frac{2 B w}{1-w^{2}}\right) \frac{2\left(1+w^{2}\right)}{\left(1-w^{2}\right)^{2}} .
$$

In order to apply the saddle point method for approximate evaluation of the contour integral (3.2) we need to find saddle points of $g(w) \hat{\Phi}_{N}(w)$. It is evident that the location of these saddle points is symmetric with respect to the real and the imaginary axes, so that we confine ourselves to the first quadrant $(\operatorname{Re} w \geqq 0, \operatorname{Im} w>0)$.

Now the saddle points of $g(w)$ are closely related to the singularities of $g(w)$, i. e. the zeros of $\cosh \left(A \sinh \frac{2 B w}{1-w^{2}}\right)$. They are nothing but the images of $z=\infty$ by (2.2), i. e. by

$$
z=\phi(w)=\tanh \left(A \sinh \frac{2 B w}{1-w^{2}}\right),
$$

and $z=\infty$ is mapped onto an infinite array of singularities which accumulate to $w=1$. Note that $w=1$ is the essential singular point of $g(w)$. Take $A=B=\frac{\pi}{2}$, for example. Then the singularities, in particular those which we are most interested in, are given as

$$
p_{k}=\left(\sqrt{B^{2}+\zeta_{k}^{2}}-B\right) / \zeta_{k}, \zeta_{k}=\frac{\pi}{2} i+\operatorname{arcosh}(2 k+1), k=0,1, \ldots
$$

in the first quadrant. The family of points $\left\{p_{k}\right\}$ corresponds to the multi-valuedness of $\operatorname{artanh}$ in $z=\operatorname{artanh}\left(\frac{\pi}{2} \sinh \zeta\right)$, i. e. to

$$
\frac{\pi}{2} \sinh \zeta_{k}=\frac{\pi}{2}(2 k+1) i, k=0,1, \ldots
$$

The principal singular points $p_{0}=i$ for $k=0$ is isolated, while the others are quite close to each other as shown in Fig. 2. Let $\Gamma$ be a smooth curve ending at $w=1$ on which all the singularities $p_{0}, p_{1}, p_{2}, \ldots$ 
lie, i. e.

$$
\Gamma:\left\{\begin{array}{l}
\omega=\left(\sqrt{B^{2}+\zeta^{2}}-B\right) / \zeta \\
\zeta=\frac{\pi}{2} i+\operatorname{arcosh}(2 t+1), 0 \leqq t<\infty
\end{array}\right.
$$

In view of the fact that $\left|\hat{\Phi}_{N}(u+i v)\right|$ given by (3.3) decreases very rapidly as $v$ increases while $g(w)$ has an array of singularities $\left\{p_{1}, p_{2}, \ldots\right\}$ accumulating to $w=1$ along $\Gamma$ and decays very rapidly as $w \rightarrow 1$ along the $u$-axis, it is intuitively clear that there exists a saddle point $s$ located on the left side of the curve $\Gamma$ in the neighborhood of $p_{1}$ or $p_{2}$.

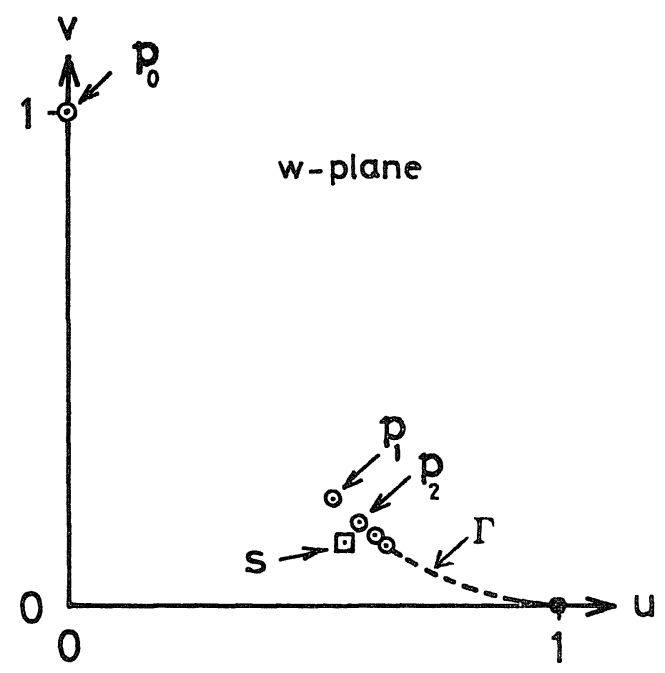

Fig. 2

In Appendix it is shown that, when $N$ is large, the saddle point $s$ is located approximately at

$$
s=1+r \exp i(\pi-\theta),
$$

where

$$
\left\{\begin{array}{l}
r=\frac{B}{\sigma}\left(1-\frac{2}{\sigma+2} \log \frac{B}{\sigma}\right) \\
\theta=\frac{\frac{\pi}{2}}{2+\frac{B}{r}},
\end{array}\right.
$$


and

$$
\sigma=\log \frac{\pi N}{A B(1+\alpha)}+\frac{B}{2}
$$

and that the intrinsic error, together with the contribution from the saddle points in the other three quadrants is expressed asymptotically as

$$
\left|\Delta I_{N}\right| \approx \frac{8 \sqrt{2} 2^{2 \alpha} \pi \sqrt{N}}{(1+\alpha) \sqrt{B}} r \exp \left(-\frac{\pi^{2} N B}{2 \sigma^{2}}\left(1+\frac{2}{\sigma+2} \log _{\bar{B}}\right)^{\frac{\sigma}{2}}\right)
$$

Take, for example, $A=B=\frac{\pi}{2}, \alpha=0, N=40$. Then we have $r \approx 0.442$ and $\theta \approx 0.283$, so that $\left|\Delta I_{N}\right| \approx 1.7 \times 10^{-9}$. The observed error, on the other hand, is $1.9 \times 10^{-9}$. When $N$ is large, $\sigma$ behaves approximately as $\log N$ and $r \approx B / \log N$, and hence the intrinsic error is given asymptotically as

$$
\left|\Delta I_{N}\right| \approx C_{1} \exp \left(-C_{2} \frac{N}{(\log N)^{2}}\right), N \rightarrow \infty .
$$

At first sight a larger value of $B$ seems to favor the present formula because then the absolute value of the exponent in (3.13) becomes larger. But it is not the case. Choose $A=\frac{\pi}{2}$ for simplicity. As $B$ becomes large, the contribution from the pole

$$
p_{0}=\frac{2}{\pi} i\left(B-\sqrt{B^{2}-\frac{\pi^{2}}{4}}\right)
$$

increases. When $B=\frac{\pi}{2}$, we see that $p_{0}=i$ and that the contribution from $p_{0}$ can be neglected, but when $B=\pi$, for example, we have $p_{0}=(2-\sqrt{3}) i$ and the value of $\left|\hat{\Phi}_{N}\left(p_{0}\right)\right|$ is quite large. Hence we see that it is not profitable to make $B$ too large and that a value around $\frac{\pi}{2}$ is suitable for $B$ if we take into account the contribution both from $s$ and from $p_{0}$.

\section{$\S 4$. Level Map of the Characteristic Function $\left|\Phi_{N}(z)\right|$}

If we transform the error integral (3.2) by means of (3.6), we 
have another form of error integral

$$
\Delta I_{N}=\frac{1}{2 \pi i} \oint_{c} \Phi_{N}(z) f(z) d z
$$

and a characteristic function expressed in terms of the variable $z$

$$
\Phi_{N}(z)=\Phi_{N}(\phi(w))=\hat{\Phi}_{N}(w) .
$$

The path $C$ is the image of $\hat{C}$ mapped by (3.6). A level map of $\left|\Phi_{N}(z)\right|$ is very useful for practical error estimation in particular when the given integrand has singularities in the finite $z$-plane other than $z= \pm 1$ [8]. We obtain a level curve corresponding to

$$
\varepsilon=\frac{1}{2 \pi}\left|\Phi_{N}(z)\right|=\frac{1}{2 \pi}\left|\hat{\Phi}_{N}(w)\right| \approx \exp (-\pi N|\operatorname{Im} w|)
$$

by drawing an image of the line

$$
|\operatorname{Im} w|=\frac{1}{\pi N} \log \frac{1}{\varepsilon}
$$

mapped by (3.6). In Fig. 3 we show a level map of $\frac{1}{2 \pi}\left|\Phi_{N}(z)\right|$ in

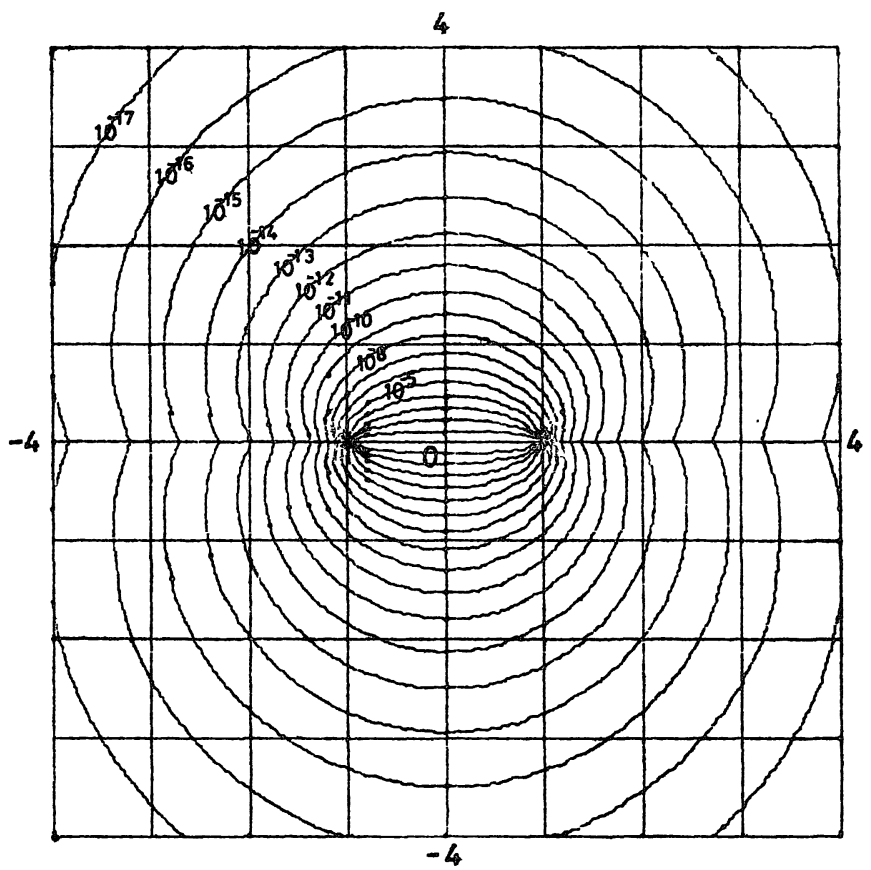

Fig. 3 The level map of $\frac{1}{2 \pi}\left|\Phi_{N}(z)\right|$ for $x=\tanh \left(\frac{\pi}{2} \sinh \frac{\pi}{2}\left(\frac{1}{1-u}-\frac{1}{1+u}\right)\right), N=32$. 
the case of $A=B=\frac{\pi}{2}$ and $N=32$.

Although the numbers specifying the curves are the values of $\frac{1}{2 \pi}\left|\Phi_{N}(z)\right|$ for $N=32$, we can also use this map for estimating errors when $N=2^{m}, m=1,2,3, \ldots$ Take a level curve of $\varepsilon=10^{-m}$, then we have

$$
|\operatorname{Im} w|=\frac{M \log 10}{\pi N},
$$

and hence, when the mesh size is halved $(N \rightarrow 2 N)$, we should specify the level curve to be of $\varepsilon=10^{-2 M}$. For example, the level curve of $10^{-16}$ in Fig. 3 should be read as of $10^{-32}$ when $N=64$, and $10^{-8}$ when $N=16$, etc.

For comparison a level map of $\frac{1}{2 \pi}\left|\Phi_{h}(z)\right|$ for the proper double exponential formula (1.10) is shown in Fig. 4. Although the numbers specifying the curves are values of $\frac{1}{2 \pi}\left|\Phi_{h}(z)\right|$ when $h=0.25$, this map can also be used for other values of $h$ provided that

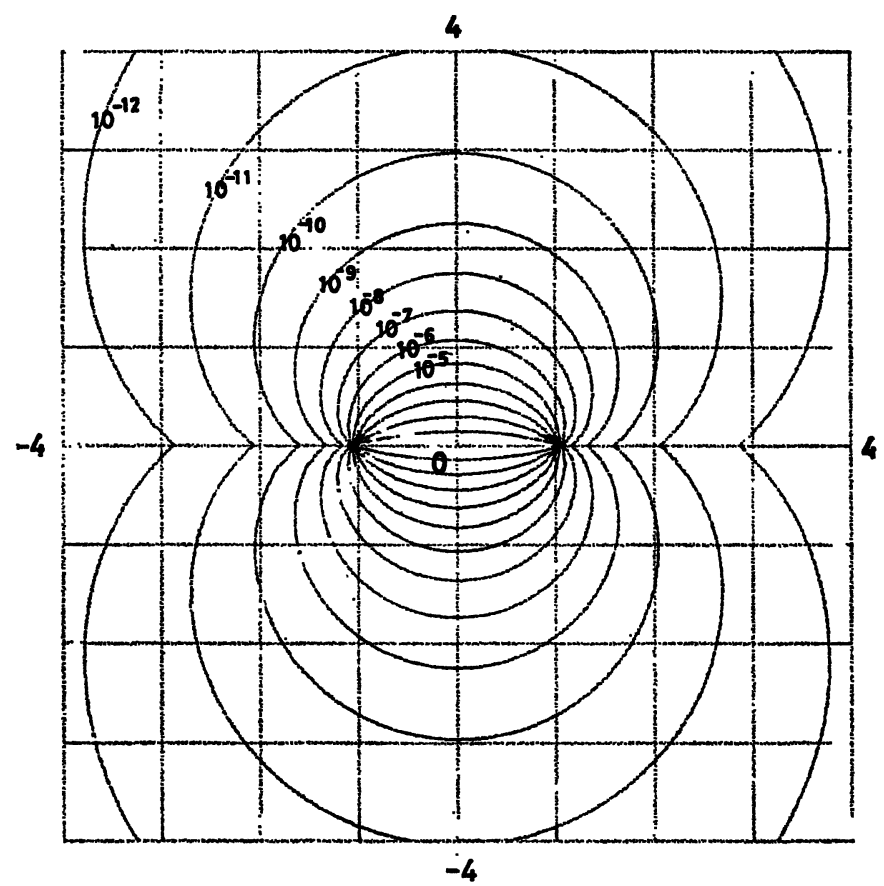

Fig. 4 The level map of $\frac{1}{2 \pi}\left|\Phi_{h}(z)\right|$ for $x=\tanh \left(\frac{\pi}{2} \sinh u\right), h=0.25$. 
$h=1 / 2^{m}, m=1,2, \ldots$

\section{§5. Numerical Examples}

In this section we shall give some numerical examples. Throughout this section we take $A=B=-\frac{\pi}{2}$ for the IMT-type double exponential formula. We also give results obtained using the double exponential formula (1.10).

The first integrand is

$$
f(x)=\left(1-x^{2}\right)^{\alpha}, \alpha=\frac{1}{2}, 0,-\frac{1}{2},
$$

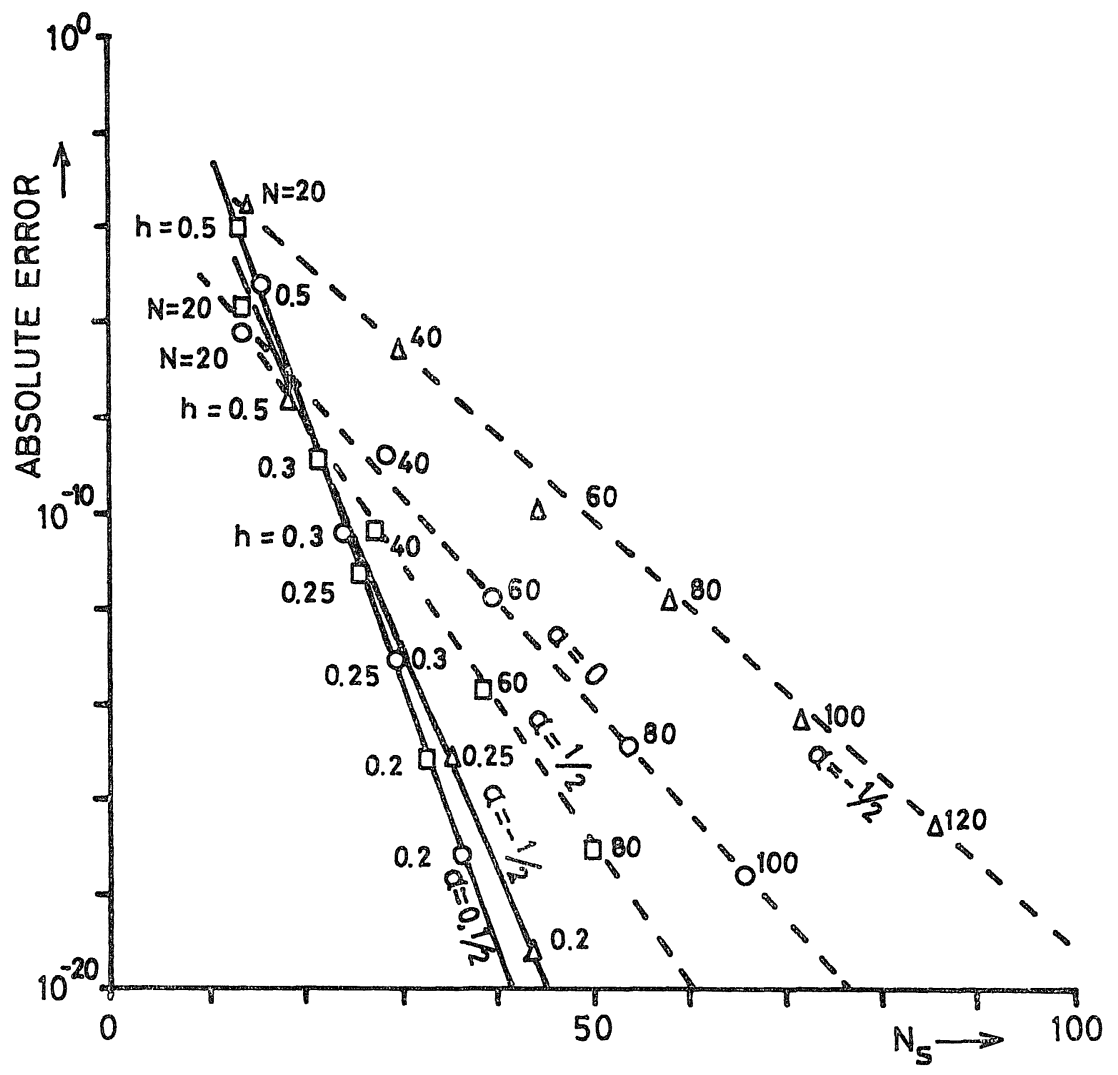

Fig. 5 Absolute errors observed when $\int_{-1}^{1}\left(1-x^{2}\right)^{\alpha} d x, \alpha=\frac{1}{2}, 0,-\frac{1}{2}$ is computed.

the double exponential formula

- - - - the IMT-type double exponential formula

$\square ; \alpha=\frac{1}{2}, \bigcirc ; \alpha=0, \triangle ; \alpha=-\frac{1}{2}$ 
and the absolute errors which we obtained by subtracting the computed value from the exact value of the integral are shown in Fig. 5. They are intrinsic errors. Since the decay of $g(u)=f(\phi(u)) \phi^{\prime}(u)$ as $u \rightarrow \pm 1$ is generally very rapid, the points which lie very close to $u= \pm 1$ are not sampled, i. e. the summation in (2.5) is truncated as

$$
\begin{aligned}
I_{N_{s}} & =\frac{2}{N} \sum_{n=M_{1}}^{M_{2}} f(\phi(-1+n h)) \phi^{\prime}(-1+n h), M_{1} \geqq 1, M_{2} \leqq N-1, \\
N_{s} & =M_{2}-M_{1}+1 .
\end{aligned}
$$

Hence the abscissas $N_{s}$ of Figs. 5, 6 and 7 are the numbers of points actually sampled in the computation. In fact, for any integrand, the truncation must be done as in (5.2) in actual computation in order to avoid overflow in the evaluation of $\phi(-1+n h)$ and $\phi^{\prime}(-1+n h)$. Moreover, in order to obtain smooth plots in Figs. 5, 6 and 7, the truncation points were adjusted carefully so that the highest precision was obtained with a minimum number of sampling points for each $h=2 / N$. The situation is the same in the case of the double exponential formula (1. 10).

In Fig. 6 we show the absolute errors observed when

$$
f(x)=\frac{1}{1+x^{2}}
$$

is integrated. The errors in this case can be estimated very easily from Figs. 3 and 4 by means of the residue theorem applied to (4. 1), i. e. by

$$
\Delta I_{N}=-\sum_{j} R_{j} \Phi_{N}\left(z_{j}\right)
$$

where $z_{j}$ is the $j$-th pole of $f(z)$ and $R_{j}$ is the corresponding residue. In the present example with $N=32$ this reduces to

$$
\begin{aligned}
\left|\Delta I_{N}\right| & =\left|\frac{1}{2} i\left(\Phi_{N}(i)-\Phi_{N}(-i)\right)\right| \leqq\left|\Phi_{N}(i)\right| \\
& \approx 2 \pi \times\left(3 \times 10^{-8}\right) \approx 2 \times 10^{-7}
\end{aligned}
$$

which agrees well with the result in Fig. 6.

Comparing Figs. 5 and 6, we see that the proper double exponential formula is better when the integrand has end point singularities, 


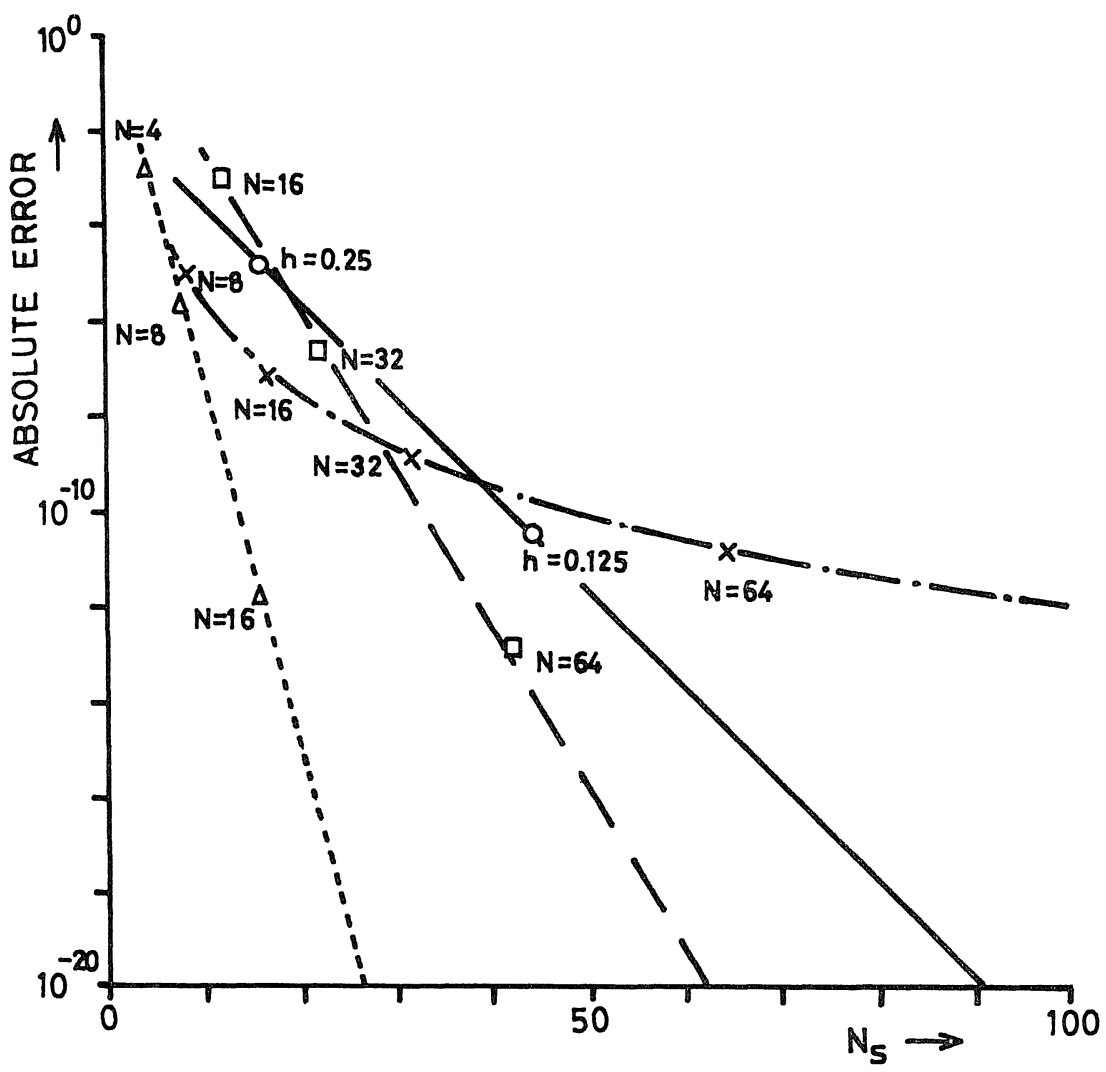

Fig. 6 Absolute errors observed when $\int_{-1}^{1} \frac{1}{1+x^{2}} d x=1.570796 .$. is computed.

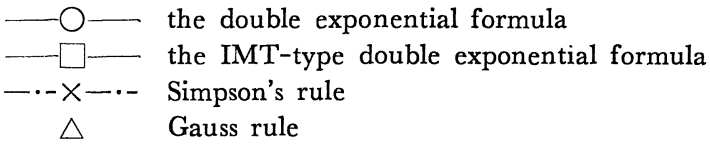

while the IMT-type double exponential formula is better when the integrand is regular at the end points. We understand this situation intuitively because the sampling points of the former are distributed more densely in the neighborhood of the end points than those of the latter. In fact, one can take as many points as one wishes for the proper double exponential formula, while the number of the points of the IMT-type one is finite. Therefore, when one integrates functions with end point singularities, one should use the proper double exponential formula. On the other hand, it is known that the Clenshaw-Curtis formula whose distribution of the sampling points is 
somewhat similar to our formulas in the sense that it is rather dense in the neighborhood of the end points $[2$, p. 68], is efficient when the integrand is regular on the interval of integration including the end points, while it is quite inefficient when the integrand has end point singularities. In conclusion, we can locate the IMT-type double exponential formula between the proper double exponential formula and the Clenshaw-Curtis formula.

Finally in Fig. 7 the absolute errors produced when

$$
f(x)=\frac{1}{(x+2)(1-x)^{3 / 4}(1+x)^{1 / 4}}
$$

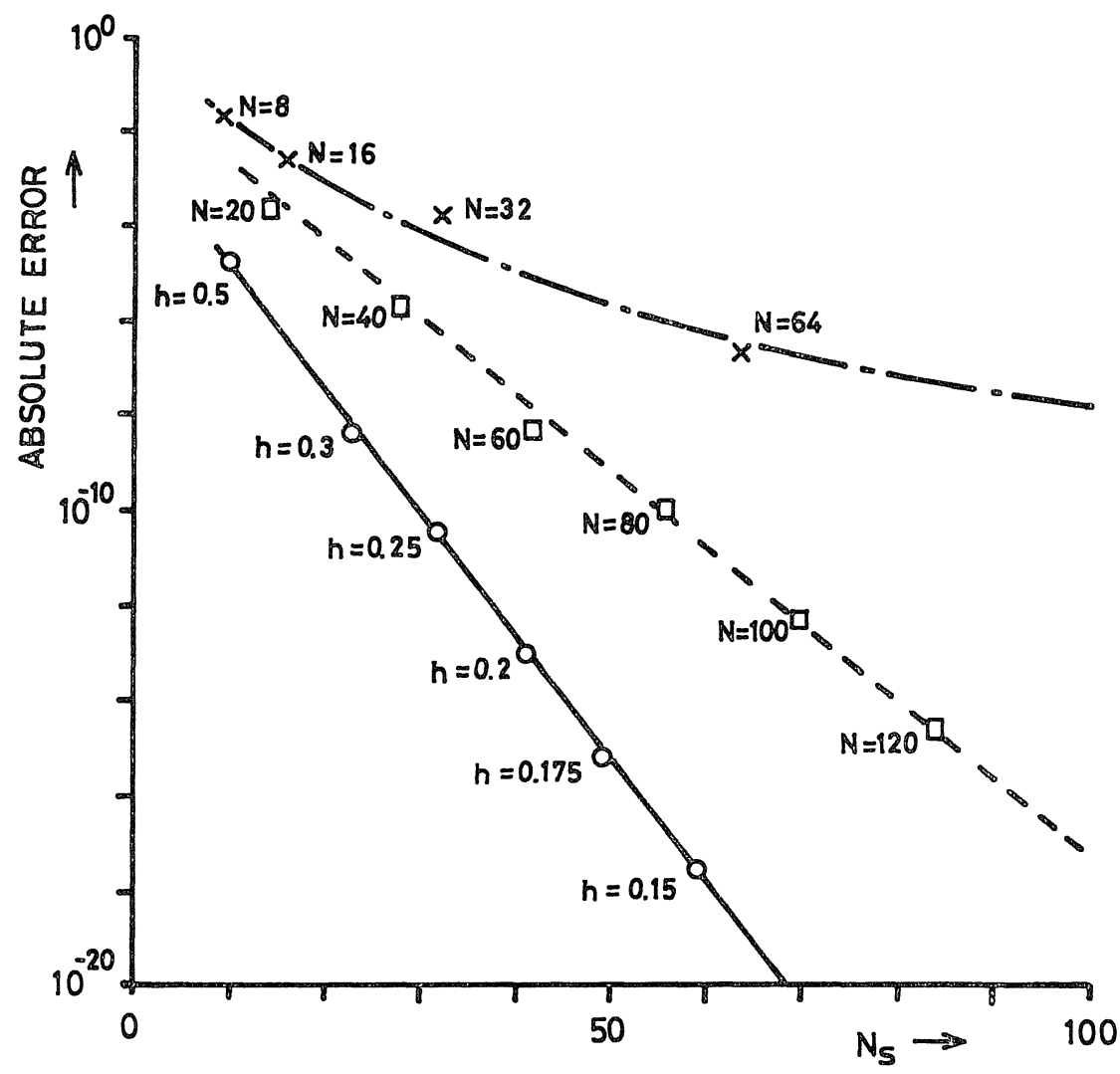

Fig. 7 Absolute errors observed when $\int_{-1}^{1} \frac{d x}{(x+2)(1-x)^{3 / 4}(1+x)^{1 / 4}}=1.9490 \ldots$ is computed.

- - - the double exponential formula

- - $\square-$ - - the IMT-type double exponential formula

$-\cdot-\times-\cdot-$ the IMT-rule 
is integrated are shown. It is seen from Fig. 4 that the contribution from the pole at $x=-2$ to the error is dominated in the case of the proper double exponential formula, while the main contribution in the case of the IMT-type double exponential formula comes from the intrinsic error.

We obtain various kinds of IMT-type double exponential formulas not only for integrals over a finite interval but also for integrals over $(0, \infty)$ or $(-\infty, \infty)$ if we replace $u$ in the proper double exponential transforms [10] by $2 B u /\left(1-u^{2}\right)$.

\section{Appendix}

In a region where the end point effect of $\hat{\Phi}_{N}(w)$ around $w=1$ can be neglected and, at the same time, the term $1 /(1+w)$ in $g(w)$ can be neglected compared with $1 /(1-w)$, we can approximate $\hat{\Phi}_{N}(w) g(w)$ as

$$
\text { (A. 1) } \quad \hat{\Phi}_{N}(w) g(w) \approx \frac{2^{2(1+\alpha)} \pi \lambda i}{(1+\alpha) B}\left(\frac{B}{1-w}\right)^{2}\left(\exp \frac{B}{1-w}\right) \exp \psi(w),
$$

where

$$
\psi(w) \equiv i \pi N w-\lambda \exp \frac{B}{1-w}
$$

and

$$
\lambda=A(1+\alpha) \exp \left(-\frac{B}{2}\right)
$$

Then the saddle point $s$ which is a solution of $\frac{d}{d w} \hat{\Phi}_{N}(w) g(w)=0$ is approximated by the corresponding solution of

$$
\psi^{\prime}(w)=i \pi N-\frac{\lambda B}{(1-w)^{2}} \exp \frac{B}{1-w}=0 .
$$

We express the solution $s$ of (A.4) in terms of the polar coordinate system as

$$
\text { (A. 5) } s=1+r \exp i(\pi-\theta), 0<\theta<\pi \text {. }
$$

Then (A. 4) reduces to 
(A. 6)

$$
\left\{\begin{array}{l}
B \lambda \exp \left(\frac{B}{r} \cos \theta\right) \cos \left(2 \theta+\frac{B}{r} \sin \theta\right)=0, \\
B \lambda \exp \left(\frac{B}{r} \cos \theta\right) \sin \left(2 \theta+\frac{B}{r} \sin \theta\right)=\pi N r^{2} .
\end{array}\right.
$$

From the first equation of (A. 6) we have

$$
\frac{B}{r} \sin \theta=\frac{\pi}{2}-2 \theta,
$$

while from the second one we have

$$
\exp \left(\frac{B}{r} \cos \theta\right)=(\exp \sigma) r^{2}
$$

where $\sigma$ is given by (3.12). Assuming that $\theta$ is small, we have from (A. 8)

$$
\frac{B}{r} \approx \sigma+2 \log r
$$

and from (A. 7)

(A. 10)

$$
\theta \approx \frac{\frac{\pi}{2}}{2+\frac{B}{r}}
$$

In order to solve (A. 9) or $\sigma-B / r+2 \log r=0$ for $r$, we first guess $r=B / \sigma$ and apply the Newton's method once. Then we have

$$
r \approx \frac{B}{\sigma}\left(1-\frac{2}{\sigma+2} \log \frac{B}{\sigma}\right)
$$

as an approximation of the radial part of the saddle point. On the other hand, we have from (A. 2)
(A. 12)

$$
\operatorname{Re} \psi(s)=-\pi N r \sin \theta-\lambda \exp \left(\frac{B}{r} \cos \theta\right) \cos \left(\frac{B}{r} \sin \theta\right) .
$$

Then by (A. 8), (A. 7) and (A. 10), $\operatorname{Re} \psi(s)$ reduces to

$$
\begin{aligned}
\operatorname{Re} \psi(s) & \approx-\pi N r \sin \theta-\frac{\pi N}{B} r^{2} \cos \left(\frac{\pi}{2}-2 \theta\right) \\
& =-\pi N r \sin \theta\left(1+\frac{2 r}{B} \cos \theta\right) \\
& \approx-\pi N r \theta\left(1+\frac{2 r}{B}\right) \approx-\frac{\pi^{2} N}{2 B} r^{2} .
\end{aligned}
$$


Since

$$
\left(\frac{B}{1-s}\right)^{2} \exp \frac{B}{1-s}=\frac{\pi B N}{\lambda} i
$$

from (A. 4), the contribution of the integral (3.2) in the neighborhood of the saddle point $s$ is given as

$$
\text { (A. 15) }|\Delta I(s)| \approx \frac{\left|\hat{\Phi}_{N}(s) g(s)\right|}{\sqrt{2 \pi\left|\phi^{\prime \prime}(s)\right|}} \approx \frac{2^{2(1+\alpha)} \pi^{2} N}{(1+\alpha) \sqrt{2 \pi\left|\phi^{\prime \prime}(s)\right|}} \exp (\operatorname{Re} \phi(s))
$$

by the formula of the saddle point method. Substituting (A. 13) and

$$
\text { (A. 16) } \quad \begin{aligned}
\phi^{\prime \prime}(s) & =-\frac{1}{1-s}\left(2+\frac{B}{1-s}\right) \frac{\lambda B}{(1-s)^{2}} \exp \frac{B}{1-s} \\
& =-\frac{1}{1-s}\left(2+\frac{B}{1-s}\right) \times i \pi N \approx-\frac{i \pi N B}{(1-s)^{2}}
\end{aligned}
$$

into (A. 15) and taking into account also the contribution from the saddle points located in the other three quadrants, we have finally

$$
\left|\Delta I_{N}\right| \approx \frac{8 \sqrt{2} 2^{2 \alpha} \pi \sqrt{N} r}{(1+\alpha) \sqrt{B}} \exp \left(-\frac{\pi^{2} N}{2 B} r^{2}\right)
$$

and (3. 13).

\section{References}

[1] Davis, P.J., On the numerical integration of periodic analytic functions, On Numerical Approximation, R. E. Langer, ed., The University of Wisconsin Press, (1959), 45-59.

[2] Davis, P. J.and Rabinowitz, P., Methods of Numerical Integration, Academic Press, 1975.

[ 3 ] Dixon, V.A., Numerical quadrature: a survey of the available algorithms, Software for Numerical Mathematics, D. J. Evans, ed., Academic Press, (1974), p. 127.

[4] Iri, M., Moriguti, S. and Takasawa, Y., On a certain quadrature formula (in Japanese), RIMS Kokyuroku Kyoto Univ., 91 (1970), 82-118.

[5] Sag, T.W. and Szekeres, G., Numerical evaluation of high-dimensional integrals, Math. Co mp., 18 (1964), 245-253.

[6] Schwartz, C., Numerical integration of analytic functions, J. Computational Phys., 4 (1969), 19-29.

[7] Stenger, F., Integration formulae based on the trapezoidal formula, J. Inst. Math. Appl., 12 (1973), 103-114.

[8] Takahasi, H. and Mori, M., Error estimation in the numerical integration of analytic functions, Rep. Comput. Centre Univ. Tokyo, 3 (1970), 41-108.

[9] Quadrature formulas obtained by variable transformation, Numer. Math., 21 (1973), 206-219.

[10] Double exponential formulas for numerical integration, Publ. RIMS, Kyoto Univ., 9 (1974), 721-741. 
